\title{
OBJECTIVES AND TREATMENT IN THE MIXED DENTITION
}

\author{
Harlow L. Shehan, D.D.S., Jackson, Mich., and \\ Edward A. Cheney, D.D.S., M.S., Lansing, Mich.
}

TT IS a privilege to discuss some of the intercsting problems which arise during 1 the mixed dentition. There are, during this stage of development, a great many opportunities to guide growth and intercept malocclusion. The problems of this period challenge the orthodontist to understand and recognize the dentofacial needs of the growing child. It is a period where treatment must be concise and often is dramatic. It is a period when we can direct the developmental potentialities of the individual, but, also, it is a period when the integrity of the practitioner is open to judgment. Whereas, in the early adult dentition he can draw conclusions based on clinical evaluations alone, here the orthodontist must also include the factor of growth and must know that the dentition has had its chance of expression before modification is initiated.

There are numerous developmental changes which take place during the mixed dentition. Many of these are expected. Just prior to the beginning of the transition from primary to permanent dentition, the face, jaws, and teeth present a most interesting combination. The face is small in proportion to the other parts of the skull. The jaws are but buccal and lingual plates. Between them lie the partially and fully developed crowns and roots of the primary and permanent teeth. At this time there are more teeth present than at any other time in the life span. Twenty primary and twenty-eight permanent dental units are closely grouped and intricately spaced. They stand poised for one of the the most complicated and exciting developmental "sprees" of the human body, the transition stage of dental development.

Broadbent graphically demonstrated the development which takes place during the mixed dentition. He carefully pointed out the flexibility which attends these changes. He demonstrated the high degree of integration between the teeth and the jaws as they work together to produce the adult dental unit. In addition, he illustrated that all is not beautiful during the changing sequences of the mixed dentition.

It is not our intention to review the sequences of dentofacial growth during the transition stage. Anyone who considers mixed dentition treatment must be well versed in the developmental changes of this period. Rather, we wish to emphasize that the growing dentition has a great potentiality to express itself to the fullest extent. Treatment procedures during the transition stage should not be initiated until full recognition of these potentialities has been given. Often the individual's ability to develop is such that correction of undesirable relation-

Presented at the Twenty-second Annual Meeting of the Great Lakes Society of Orthodontists, Cleveland, Ohio, Nov. 5, 6, and 7, 1951.

From the Department of Orthodontics, University of Michigan. 
ships will occur as growth takes over in its orderly form. We have no license to step in and treat where the developing dentition has the potentiality to overcome irregularities of its own volition.

For example, consider the problem of lower crowding during the early mixed dentition. Often, much to the concern of the parents and dentists, the permanent incisors erupt lingual to the lower primary incisors and are crowded. As illustrated in Fig. 1, irregularities of this type often straighten out as growth proceeds. In this illustration there are six consecutive views of lower casts taken of the same individual one year apart. In Fig. $1, A$ and $B$ the permanent central incisors have erupted to the lingual and have moved forward. In Fig. 1, $C$ and $D$ the permanent lateral incisors have erupted lingually to their primary predecessors. This is to be expected, since they also develop in this relationship. Here, even though the primary lateral incisors were not extracted, they shortly exfoliated and in one year the permanent successors moved into position. The same course of development was followed by the canines in Fig. 1, $E$ and $F$.

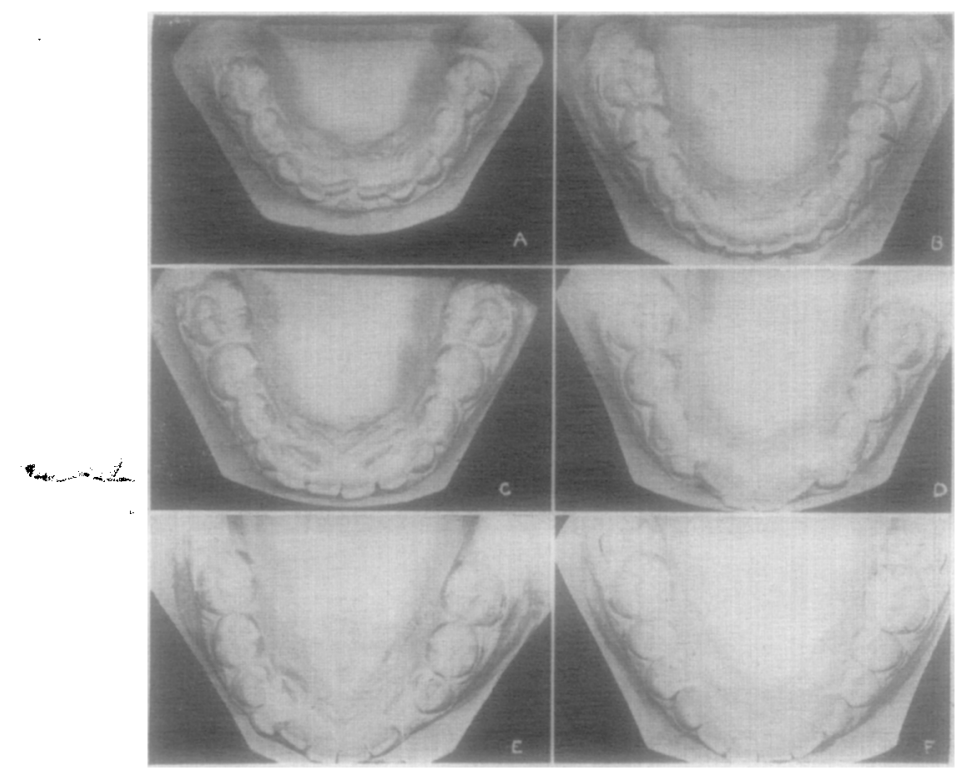

Fig. 1 .

There are many growth sequences similar to the one just illustrated that are typical of normal growth changes which take place during the mixed dentition. The spacing and closing of maxillary central and lateral incisors, the slow ramblings of maxillary canines, and the shifting of premolars are others. There is no doubt that many of these changes do not coordinate as well as the one just illustrated. Yet in no case can any of them be termed abnormal or requiring treatment until sufficient observational data indicate that the sequence is out of order.

The extraordinary ability of the developing dentition to unravel many of its own idiosyncrasies is even more dramatically illustrated in Fig. 2. Here are the records taken of an untreated case over three successive years. The upper 
cast reveals the presence of a maxillary canine locked in $\mathrm{x}$-bite. The middle cast shows the occlusal changes incident to the transition from primary to permanent teeth. In addition the canine is changing position. The lower cast reveals that the canine has moved of its own free will. It has changed from its lingually locked position to its now correct position. This it did by itself. Ordinarily we would not expect this change. Yet this case does confirm the fact that the power of the growing dentition to help itself is exceptional. Any consideration of treatment during the mixed dentition must take account of this fact and be planned to accommodate it.

There are, however, a considerable number of developmental irregularities occurring during the mixed dentition. These warrant consideration. In our opinion, the modification of these irregularities constitutes treatment objectives, inasmuch as the removal of them not only results in a corrected relationship, but also in a change which contributes in a large proportion to a satisfactory and functional adult dentofacial complex. There is no doubt that the significance attending the presence of developmental irregularities in the mixed dentition varies from one individual to another. Yet many of those which appear most simple are often of greatest importance. We would like to review a number of conditions which in our opinion constitute developmental irregularities and discuss methods for dealing with them.

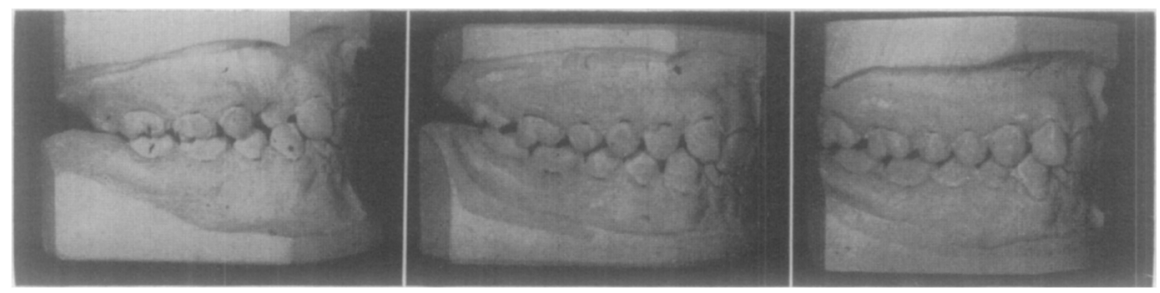

Fig. 2.

One of the most disturbing developmental irregularities which occur in the mixed dentition is the cross-bite. Cross-bites occur commonly in the anterior and posterior dentition and may involve single or several teeth at one time. They are objectionable because: (1) they may act to destroy teeth and supporting structures, (2) they may result in maldistribution of supporting alveolar bone, and (3) they may cause obvious and objectionable facial asymmetries. There are many methods of dealing with undesirable cross-bites in the developing dentition. As we have previously indicated it is our opinion that these methods must be sufficiently flexible to fit the changes and adaptation which take place within the dentition during the process of growth. In any case the appliance should be simple within the limits of simplicity permitted by the individual situation. There are several appliances which meet these requirements and which are well adapted for dealing with anterior cross-bites. One is the inclined plane. The tongue blade is a device particularly useful in correcting cross-bite relationships of central and lateral incisors. Under ideal conditions bilateral inlockings 
may be dealt with by means of the tongue blade. Usually, however, this technique is most effective when applied to cross-bites involving only one tooth in either arch.

The application of the tongue blade technique is illustrated in Fig. 3. This illustration shows a left central incisor locked lingual to its opponents. All other teeth are fairly well aligned. The patient is instructed to catch only the tip end of the blade under the incisor edge of the tooth. This relationship is shown in the upper right exposure. Often it is necessary to use narrow blades in order to avoid contact with adjacent teeth. Wide blades may be split in half for this purpose. During exercise the patient is asked to draw the blade back hard against his chin, as illustrated, always keeping the tongue blade parallel to the long axis of the tooth to be moved. The tongue blade then provides a steep inclined plane which directs the tooth labially. There is little or no intrusive force acting to depress the tooth. This consideration of intrusion is of particular importance in cases where the depth of bite is shallow. Here a small amount of intrusive action can reduce the retentive advantage of an overbite relationship of the incisor teeth and contribute to a relapse following the end of treatment.

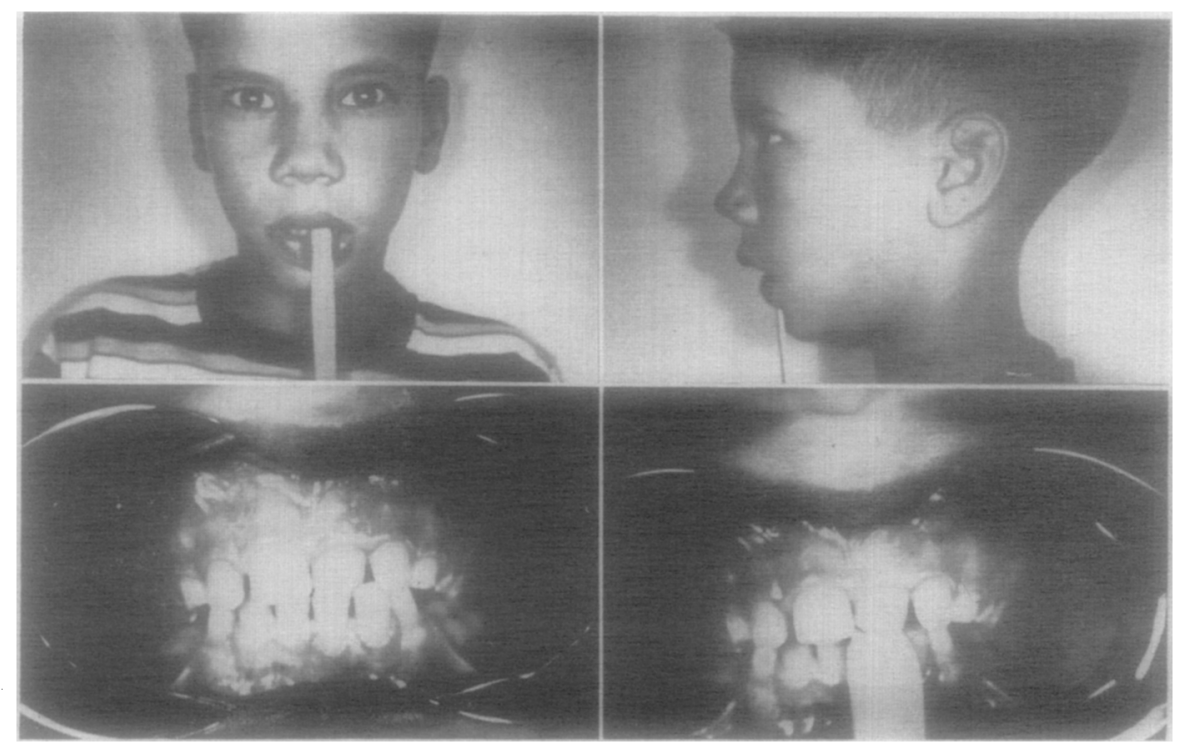

Fig. 3 .

There are many methods of exercising with the tongue blade in an effort to "jump" a lingually locked central or lateral incisor into correct position. Whatever procedure is used, it is best to be very specific when giving instructions to the patient. One successful set of instructions advises the patient to bite hard upon the plane for five counts, relax for three counts, and engage in this cxcreise to the extent of fifty contractions and relaxations of the jaws and musculature before each meal and before retiring. During the exercise the patient should main- 
tain a continuous biting contact with the tongue blade. A good way to explain this aspect of treatment is to advise him "never to take his teeth apart." This confines treatment to the area under consideration.

The exercise before each meal is of particular advantage. It enables the mother to supervise the tongue blade activity at regular periods. After a few days of hard exercise, the patient will find that he can bite with the teeth in an edge-to-edge relationship and can usually establish a slight amount of overbite. At this stage of treatment the mastication of food with the teeth in their correct relationship is of great value. It provides an acceptable reason for conlinuing the exercise and sets up the first stage of the retentive phase of treatment. Once the patient can attain an overbite relationship, he should realize that the responsibility of maintaining this change is entirely his. This fact must be thoroughly emphasized at the beginning of treatment. In most cases a conscious effort on the part of the patient to maintain the correction will bring the treatment to a successful end in a surprisingly short period of time. The treatment with the tongue blade illustrates the principles of the inclined plane.

The Hawley incline, the interdental ligation technique, and the plastic and metal inclines cemented to the lower incisors may also be used. We wish to point out that the tongue blade and similar appliances enable us to deal simply with minor growth problems at this stage of development.

As we resort to these methods, however, we must give important consideration to patient cooperation. A consideration of patient cooperation is of major importance in the use of the simpler preventive and palliative appliances. Like the tongue blade many of these devices are not fixed in nature. Accordingly, the success with the use of them is greatly dependent upon the patient's willingness to work as directed. It is up to the operator then to solicit the upmost in cooperation if he is to achieve the desired end in treatment procedures. In most cases, the solicitation of patient cooperation is neither difficult nor time-consuming. A majority of children respond graciously once they understand the nature of the problem and the reason for making the correction. Even so, however, the approach to treatment cannot be lackadaisical. You cannot just "toss" the tongue blade or any other appliance at a patient and expect results. Each individual must thoroughly realize the.limitations and abilities of the appliance of choice and know exactly what his responsibility in treatment should be. Failure on the part of the operator to present this information and failure of the patient to understand it lead to a mutual lack of confidence and add nothing to the luster and prestige of orthodontics. On the other hand, the successful use of simple treatment devices calls for an effort which challenges the best in the practitioner to gain full cooperation of the individual patient and demonstrates to the public a real understanding of the interesting biology of denotofacial development.

Posterior $\mathrm{x}$-bites are most objectionable when they result in facial asymmetries. The mere malposition of the posterior teeth themselves is not always objectionable. Usually, the relationship is not esthetically undesirable and the occlusion can be ground to rule out traumatic interference. On the other hand, in the mixed dentition there may be other implications. For example, when the 
maxillary dental arch is constricted the free-moving mandible cannot adapt to the functional centric occlusion. For this reason the patient learns to shift the jaw laterally to accommodate ocelusion during mastication. The shift creates a facial asymmetry in the form of a lateral displacement. The end result is a posterior cross-bite. Treatment in these cases calls for widening of the maxillary dental arch until the mandibular dental arch can ocelude in the usual midline functional centric relationship.

It seems important, again, to emphasize the extent to which treatment must be carried in the mixed dentition. At this stage dentofacial development is far from complete. It is only beginning to show in its final form. The modification of dental structures during this stage, then, cannot always be considered an end in itself. Treatment need be only those procedures necessary to give direction and guidance to the growing structures toward desirable relationships in the permanent dentition. Once the correct direction of growth is established, active treatment should be discontinued. The case, however, should continue to be observed until the dentition is erupted and the dentofacial relationships are well established. In the interception of cross-bite, then, as in all other preventive orthodontic procedures in the mixed dentition, a minimum of treatment is indicated for the guidance of growth.

The removal of habits when they interfere with dental development is another objective of mixed dentition treatment. 'There is much discussion about the influence of oral habits upon the developing dentition. We do not wish to enter into these controversies at this time. It is sufficient to point out that for some children engaging in oral habits no undesirable results will take place. In others the most unexpected changes will oceur. In our analysis and approach to these problems, then, we are obligated to look at the individual in order to determine what his problem is and what it means to him. When we note that habits are interfering with the growing structures we ean determine the significance of these structures and act accordingly. In some cases it is advisable to interrupt the activity. In others it will be more appropriate to let well enough alone.

The most effective approach to oral habit control is the psychological one. Habits are an integral part of every life and we hope only to replace undesirable habits with useful habits which, from our point of view, do not disturb the developing dentition. This objective can be accomplished in a number of ways. Most effective, but not necessarily easy, is the direct appeal to the patient himself. Results obtained by this approach are illustrated in Fig. 4 . The cast on the left shows the extent of the irregularity. This patient is $81 / 2$ years old. She had a neutroclusion with an obvious open-bite. A persistent finger-sucking habit prevented the incisors from erupting. The mother had not been able to cope with the habit.

Before considering the significance of the habit with the child, the mother was asked to leave the operating room. The child was shown a set of casts demonstrating that continued finger-sucking had created an unsightly irregularity. She was also allowed to examine the models of an ideal or normal occlusion. By her own examination she was able to see the implication of finger-sucking. 
In this case the child appeared willing to try. She was further instructed to work the problem out with her doctor and without the help of her mother and father.

The idea of "working the problem out with her doctor" requires also that the orthodontist gives cooperation. In this case the doctor talked with the patient every two weeks on the telephone. He showed a continuing interest in the patient's progress. Results were forthcoming. The right cast shows the change after six months' effort on the part of the patient to stop her finger habit. Apparently, the interest of an important outside member of the family was effective in soliciting cooperation in this child. The child stopped the habit. As the casts show, the results were gratifying.

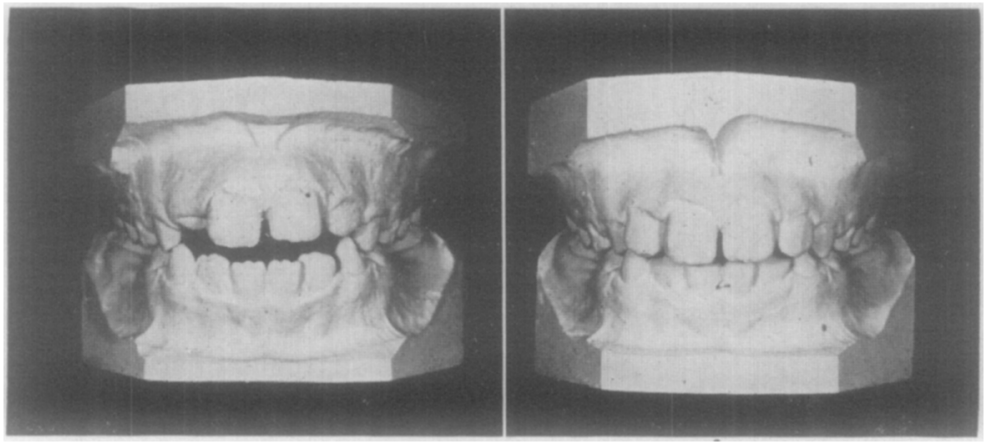

Fig. 4.

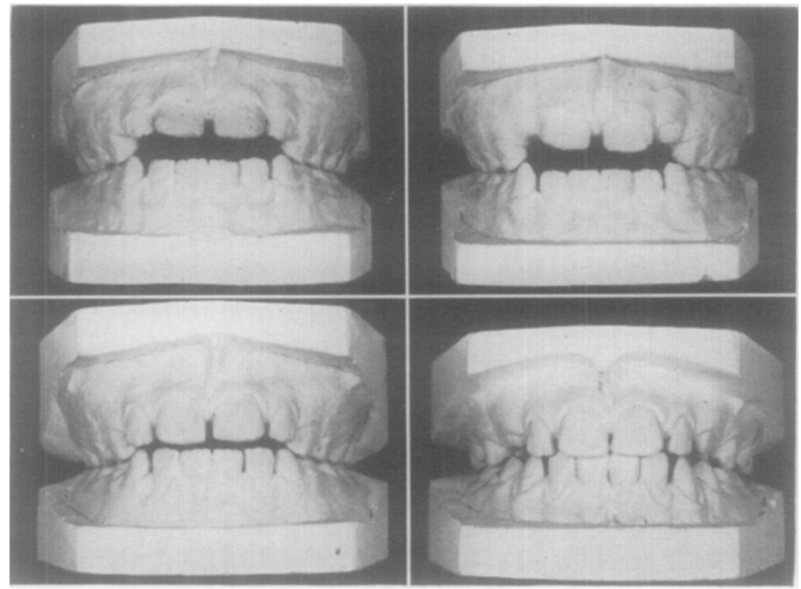

Fig. 5.

Now obviously not all children will respond to the direct appeal. Many do not seem to be able to motivate themselves along these lines. These children are often aided by "reminder devices" which serve to help them to remain aware of the habit activity. The young girl whose casts are shown in Fig. 5 needed this sort of assistance. These casts show a Class I open-bite with maxillary incisors in marked infraversion. Originally the patient had been a thumb-sucker. After 
stopping this activity, at her mother's insistence, she changed to a habit of rolling the upper portion of the tongue against the lingual surface of the maxillary incisors. Here, again, the nature and significance of the habit were explained to the patient. She was asked to put her tongue in the "bottom" of her mouth and was directly appealed to for cooperation every few weeks. However, as shown by the upper right cast no changes occurred. The patient admitted that she could not remember. With her permission we placed the reminder device shown in the center exposure. It is a plain lingual wire with short spurs soldered lingual to the maxillary incisors. For a few days the patient's tongue was highly irritated. However, she agreed to wear the device longer, as she explained to "teach her tongue a lesson." After the initial placement the patient wore this device for six months. The cast taken at that time is shown on the lower left and reveals great improvement. The appliance was removed and records taken six months later, lower right, indicate a return to her normal developmental plan.

Some children interfere with the development of their dentitions by placing fingers or blankets between their teeth during sleep. These cases are difficult to manage. Sometimes we can use lip seals to prevent the placement of offending objects in the mouth during the night. Scotch tape is one material which can be used as a lip seal. The vermilion borders of the lips are brought tightly together and are sealed together with long strips of tape which erisscross over the mouth from the infraorbital area to beneath the chin. In other cases we wish to retrude incisors which have been modified by lip or finger habit. If the incisors protrude the use of an oral shield along with the lip seals is very effective. The oral shield is made of gutta-percha or Plexiglas and is made to fit the oral vestibule. The patient's lips are brought tightly together and sealed shut over the shield. The pressure of the appliance against the incisors, during sleep, usually retrudes these teeth to a desired position. In addition, in the case of lip habits, the continuous stretching of the lips serves to increase the lip tonus and function. This activity is highly beneficial to dental development.

In the late stages of the mixed dentition we often see patients who have marked dentofacial deformities from viscous oral habits. These cases call for corrective efforts as well as habit control. They are among the most difficult to manage and often the damage is too extensive to overcome completely. Here treatment is not at all standardized. Moreover, considerations of this nature are geared to modifications typical of early adult dentition treatment. Accordingly we do not wish to consider the management of these problems. Rather we would stress that the control of undesirable oral habits is a most important early mixed dentition treatment objective. When indicated, there is no finer service which we can offer to a child.

During the mixed dentition much attention must also be given to the problems of space management. There are a great number of these cases. Basically they are problems of timing. Sometimes nature appears to confuse the rates of resorption, exfoliation, and eruption. In those situations the orthodontist has an opportunity to manage the exchange of dentitions to the best interest of the patient. The too long retention of primary teeth is one 

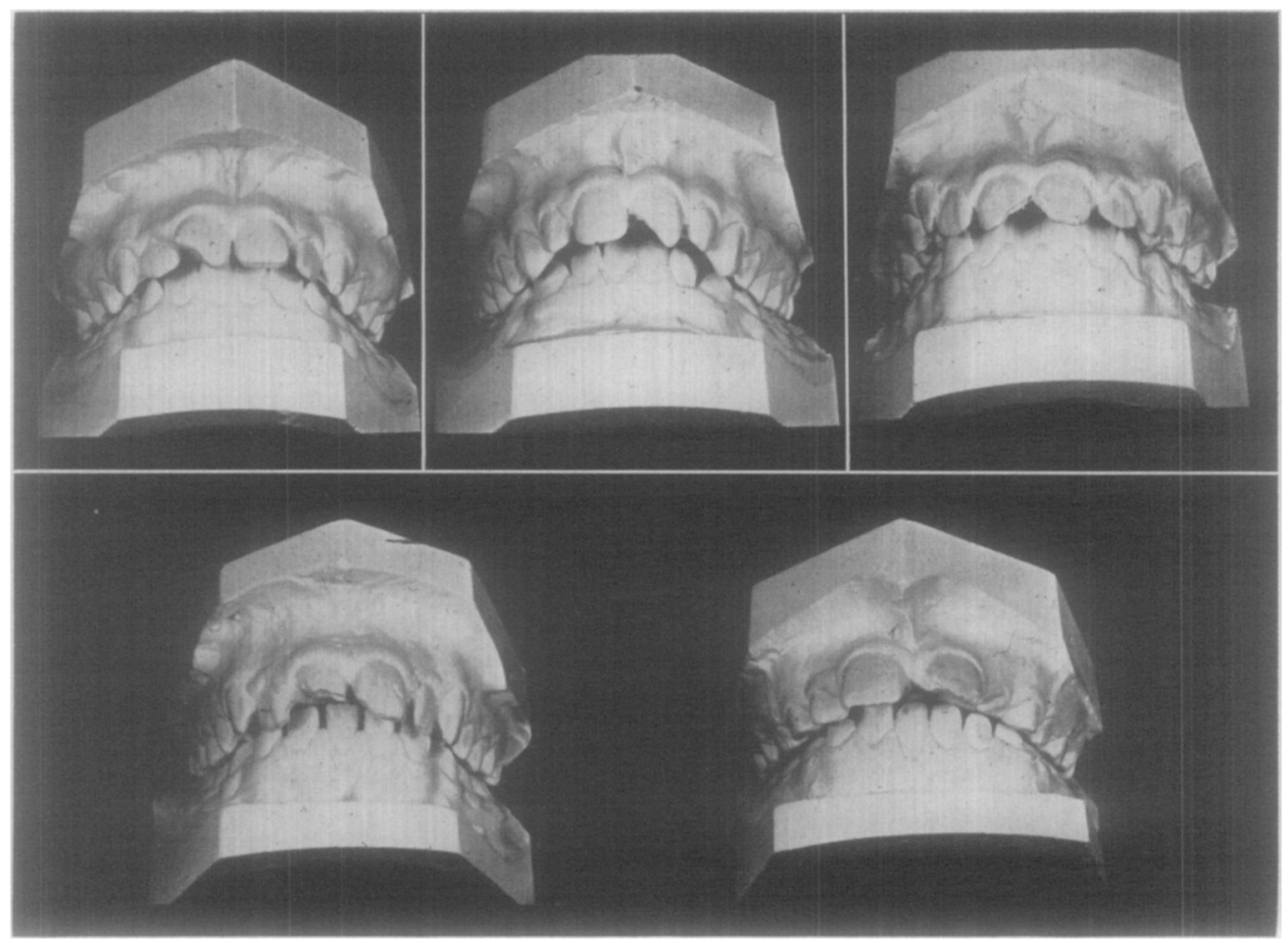

Fig. 6.

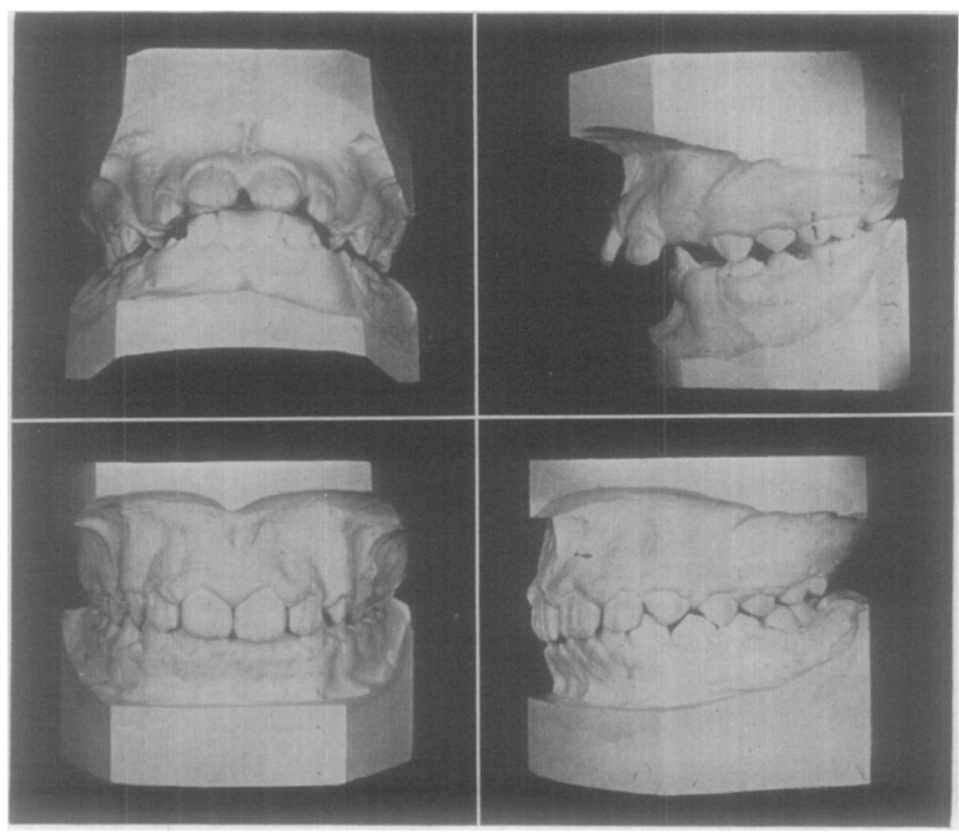

Fig. 7. 
example of these problems. As is commonly known, too long retention of primary incisors results in lingual eruption of permanent incisors. Often they grow into cross-bite relationships. The too long retention of primary molars, similarly, can result in the deflection of premolars buccally or lingually. Sometimes the primary molars oceupy too much space and the premolars are forced mesially or distally. If they happen to lock, intercuspally, in occlusion they hold these positions and cause the adjacent teeth to erupt into malposition. It is unfortunate that manay of these cases are not under supervision when the developmental crises peculiar to them arise. Often the timely removal of primary teeth and the judicious disking of the marginal ridges of primary molars serve to straighten out a disorderly growth sequence.

A great number of problems arise, also, with the early loss of primary and permanent teeth during the transition stages of the mixed dentition. Ayain treatment procedures considered in these situations must be conceived with full awareness of the growth potentialities of the individual. Close observation serves to determine these possibilities. Often, the careful collection of observational data will give us the key to the type of management needed. Many times on the basis of facts taken from the individual we learn that the use of space maintainers or mirlor dental adjustments is not needed, or should be delayed until a later time. Indeed, it seems to us that in far too many cases the mass application of space maintenance has superseded a study of the needs of the individual.

There is one other treatment objective which we would like to consider at this time. We refer to those Class II, Division 1 malocclusions in which there is extreme protrusion of the maxillary anterior teeth.

In discussing the extreme protrusions, we wish to differentiate it from those protrusions resulting from oral habits. Although habits are often assoeiated with Class II malocelusions, the protrusion in these cases is as often a disfigurement resulting from a dentofacial dysharmony as from the habit itself. Basically treatment is initiated in order to retrude the maxillary incisors. It is not habit control.

There are several reasons why we advocate the correction of extreme Class II, Division 1 protrusions as early as possible. Initially, we wish to avoid fractures to the maxillary incisors. These teeth in their protruded state are exceptionally vulnerable to any blow which is aimed in their direction. All of us have seen cases of fractured incisors. A series of these results are shown in Fig. 6. Due to accidents in play, in automobile encounters, in falling down steps, and numerous other circumstances, these and many other incisors are deformed. We have had the sad experience of advising against early treatment in these cases and then examining the patient several years later when he would return with a fractured tooth. These were unhappy occasions both for us and for the parents. Accordingly, we now advocate the treatment of all extreme protrusions in order to remove these teeth from the obvious path of trauma during the period of the mixed dentition.

These extreme protrusions are also highly undesirable from an esthetic and psychological point of view. Orthodontics is a service primarily devoted 
to the highly important responsibility of human facial appearance. The betterment of facial form is indicated at any time when it is undesirable to the patient. Many children become extremely sensitive about their teeth during the mixed dentition. The protruding incisors are often called "buck leeth" and the child is "chided" by his playmates.

Numerous cases of personality conflicts, poor schoolwork, lack of interest in playing with other children, and general resentfulness are directly associated with extreme protrusions. As shown in Fig. 7 the reduction of incisor prominence greatly enhances both the appearance and function of the developing mixed dentition. Also, as demonstrated by comparing the before and after side views in this case, there can be great advantage to the general dentofacial growth by the early correction of this distoclusion. This consideration is not a part of our discussion. However, we would like to point out that in our experience, corrections of this nature have been permanent and stable in numerous cases. Moreover, they have resulted from shorter periods of active treatment than would have been anticipated for a similar attempt in the young permanent dentition.

It has also been our experience that facial form benefits from early correction of the extreme Class II, Division 1 protrusion cases. Both by inherited inclination and by lack of function, the upper and lower lips are ineffective in stimulating dental development and in providing appearance. The upper lip stays short and curls above the protruded incisors. The lower lip is depressed, thickened, and becomes flabby beneath protruded crowns.

The retrusion of the incisors and the enabling of lip contact and closure are a worth-while contribution to dental development and facial appearance. When these changes are initiated and instigated under reasonable circumstances, they become worth-while orthodontic treatment objectives during the period of the mixed dentition.

In conclusion, there are many objectives and treatment procedures which warrant consideration in a discussion about the mixed dentition. It is our belief that these considerations should be kept to a minimum. In the mixed dentition the pattern of development, although fixed in nature, is incomplete. In general, comprehensive treatment initiated during this stage of development is not self-sustaining and is often undesirable. Many times the retentive stage of treatment is drawn out and is difficult to manage. Often the treatment becomes long and burdensome, and the financial load heavy for the family. Accordingly, except when the nature of the dentofacial complex indicates such procedure, we advocate minimum treatment efforts. For this reason our discussion has emphasized simple and short-term treatment. We advocate only those procedures necessary to guide the developing dentition and to promote its health and welfare. 